\title{
HIT000218960 promotes gastric cancer cell proliferation and migration through upregulation of HMGA2 expression
}

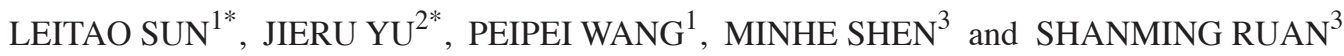 \\ ${ }^{1}$ First Clinical Medical College; ${ }^{2}$ College of Basic Medical Science, Zhejiang Chinese Medicine University; \\ ${ }^{3}$ Department of Medical Oncology, The First Affiliated Hospital of Zhejiang Chinese Medical University, \\ Hangzhou, Zhejiang 310006, P.R. China
}

Received July 6, 2018; Accepted January 31, 2019

DOI: $10.3892 / 01.2019 .10176$

\begin{abstract}
The aim of the present study was to elucidate whether the long non-coding RNA (IncRNA) HIT000218960 could accelerate the proliferative and migratory ability of gastric cancer (GC) cells by regulating high-mobility group AT-hook 2 (HMGA2) gene. The reverse transcription-quantitative polymerase chain reaction was used to determine HIT000218960 and HMGA2 expression levels in $\mathrm{GC}$ tissues and cells. The HMGA2 protein level was detected by western blotting. A $\chi^{2}$ test was used to determine the association between the HIT000218960 expression level and the clinical characteristics of patients with GC. GC cells were transfected with small interfering (si)-negative control, si-HIT000218960 and si-HIT000218960+pcDNA-HMGA2, prior to assessing the cell proliferative and migratory ability using the Cell Counting Kit-8 and Transwell assays, respectively. HIT000218960 and HMGA2 were highly expressed in GC tissues compared with in healthy tissues. In addition, HIT000218960 and HMGA2 were positively correlated in GC tissues. The HIT000218960 expression level was associated with tumor size, Tumor-Node-Metastasis staging and lymph node metastasis in patients with GC. HIT000218960 silencing decreased the proliferative and migratory ability of HGC27 and NCI-N87 cells; however, HMGA2 overexpression partly reversed this inhibitory effect. The results of the present study indicated that HIT000218960 could promote HGC27 and NCI-N87 cell proliferation and migration, which may be mediated by HMGA2.
\end{abstract}

Correspondence to: Dr Shanming Ruan, Department of Medical Oncology, The First Affiliated Hospital of Zhejiang Chinese Medical University, 54 Youdian Road, Shangcheng, Hangzhou, Zhejiang 310006, P.R. China

E-mail: shanmingruan@zcmu.edu.cn

${ }^{*}$ Contributed equally

Key words: gastric cancer, long non-coding RNA HIT000218960, high-mobility group AT-hook 2, proliferation, migration

\section{Introduction}

Gastric cancer (GC) is a malignancy that originates from the glandular epithelial cells of gastric mucosa. It is the second most common cause of cancer-associated mortality in China (1). According to the International Agency for Research on Cancer (https://www.iarc.fr/), GC was the fifth most common type of cancer worldwide in 2012 with 951,000 new cases identified, and represented the third most common causes of cancer-associated mortality, with 723,000 cases. In addition, $>70 \%$ of new cases of GC occur in developing countries, and $\sim 50 \%$ originate from Eastern Asia, mainly China (2). Treatment of GC essentially comprises surgery, chemotherapy and radiotherapy. However, the high rate of GC spreading to local lymph nodes, liver and peritoneal cavity contributes to a poor prognosis and a low 5-year survival rate $(<30 \%)(3,4)$. Numerous studies suggest that the biological evolution of GC is complex. In China, GC is typically diagnosed at an advanced stage. It is therefore crucial to improve early diagnosis of $\mathrm{GC}$ and determine its underlying molecular mechanism in order to increase the 5-year survival rate and improve the quality of life of patients with GC $(5,6)$.

Long non-coding RNA (lncRNA) is an RNA molecule of $>200$ nucleotides, which is not translated into protein (7). LncRNA was originally considered to be 'noise' in genome transcription. However, an increasing number of lncRNAs have been identified to serve a pivotal role in cancer development (8). A previous study (9) reported that lncRNA acts as a potential therapeutic target, which provides a novel direction for treatment development. Other studies $(10,11)$ have revealed that IncRNAs are associated with stomach cancer development. A previous study identified that GACAT1 expression is correlated with tumor stage, metastasis, differentiation and invasion depth of GC (12). Yang et al (13) reported that $\mathrm{H} 19$ is overexpressed in GC and correlated with cancer cell proliferation and apoptosis. In addition, H19 interacts with p53 to suppress its activity, which leads to downregulation of the downstream Bax gene, and therefore results in increased cell proliferation and decreased cell apoptosis (13). Colon cancer-associated transcript 1 (CCAT1), which is overexpressed in GC, is associated with tumor size and lymphatic or distant metastasis $(13,14)$. In vitro studies indicated that CCAT1 is associated with stomach carcinoma cell migration, 
which may result from c-Myc and E-box interaction (15). However, the expression profile and underlying mechanism of IncRNA in GC remain unclear. It is therefore crucial to investigate further the functions and underlying molecular mechanism of lncRNAs in GC.

High-mobility group AT-hook 2 (HMGA2) is a crucial protein, which is highly expressed during embryonic development and responsible for cell differentiation (16). The gene coding for HMGA2 is located on loci 13-15 of the long arm of chromosome 12 (17). Previous studies have reported that HMGA2 is overexpressed in various types of cancer, including thyroid (17), colon (18), non-small cell lung (19), squamous cell (20) and gastric (21) cancer, and is associated with invasion and metastasis of certain types of malignancy. Furthermore, HMGA2 is an independent factor that can predict the prognosis of certain malignant tumors (20). As a structural transcriptional regulator, HMGA2 is involved in numerous biological processes. For example, HMGA2 mediates glioblastoma cancer stem cell proliferation via forkhead box M1 and plasminogen activator urokinase regulation (22). In addition, HMGA2 serves a role in DNA damage repair $(23,24)$. In addition, previous studies identified that HMGA2 promotes the epithelial-mesenchymal transition (EMT) by targeting Twist family basic helix-loop-helix transcription factor 1 (Twist1), Snail family transcriptional repressor 2 and $\mathrm{Wnt} / \beta$ signaling pathways in tumors (25-28). In summary, HMGA2 serves important roles in the biological evolution of cancer. HIT000218960 is an IncRNA which has not been extensively investigated. A recent study identified that HMGA2 regulates HIT000218960 expression in thyroid carcinoma tissues (29); however, the association between HMGA2 and HIT000218960 in GC remains unclear.

To the best of our knowledge, the present study is the first to investigate the role of HIT000218960 and HMGA2 in GC. The present study clarified the regulatory effects of HIT000218960 and HMGA2 on cell proliferation and migration in gastric tumor, which may provide a novel strategy and potential target for the treatment of GC.

\section{Materials and methods}

Tissue samples and clinical characteristics of patients with $G C$. In total, 60 tumor samples were obtained from patients with GC who were admitted to The First Affiliated Hospital of Zhejiang Chinese Medical University (Hangzhou, China) between March 2016 and March 2017. A total of 25 normal tissues adjacent to cancer were selected as controls. All fresh tissues were quickly frozen in liquid nitrogen. The clinical characteristics of patients with GC were collected, including age, sex, tumor size, Tumor-Node-Metastasis (TNM) staging and lymph node metastasis. The present study was approved by the Ethics Committee of The First Affiliated Hospital of Zhejiang Chinese Medical University. All subjects provided written informed consent.

Cell culture and transfection. The HGC27 and NCI-N87 cell lines (purchased from Shanghai Huiying Biotechnology Co., Ltd., Shanghai, China), which are commonly used as GC cell lines, were selected for the present study $(30,31)$. HGC27 and NCI-N87 cells were cultured in RPMI-1640 (HyClone; GE Healthcare Life Sciences, Logan, UT, USA) containing 10\% fetal bovine serum (FBS) (Gibco; Thermo Fisher Scientific, Inc., Waltham, MA, USA) and placed at $37^{\circ} \mathrm{C}$ in a humidified incubator containing $5 \% \mathrm{CO}_{2}$. Culture medium was changed after 2 days and replaced according to the cell culture conditions. Cell lines were transfected with small interfering (si)-negative control (NC) (5'-UCCGGCGACCGGGUAACC UTT-3'), si-HIT000218960 (5'-CCUAACCCAGAGCUCUGA UUATT-3') and si-HIT000218960+pcDNA-HMGA2, which were designed and synthesized by GenePharma Biotech Corp., Taipei, Taiwan. A total of $5 \mu \mathrm{l}$ si-NC, si-HIT000218960 and pcDNA-HMGA2 were dissolved in $250 \mu 1$ transfection dilution solution, containing LipoHigh transfection reagent (catalog no. E607403; Sangon Biotech Co., Ltd., Shangai China). The mixture was incubated at room temperature for $20 \mathrm{~min}$. The cells were washed three times with PBS prior to transfection and the PBS was then replaced with fresh serum-free culture medium. The cells were incubated with the transfection mixture at $37^{\circ} \mathrm{C}$ in a $5 \% \mathrm{CO}_{2}$ incubator and the medium was changed $6 \mathrm{~h}$ post-transfection.

Reverse transcription-quantitative polymerase chain reaction (RT-qPCR). Total RNA was isolated from tissues samples and cancer cells using TRIzol ${ }^{\circledR}$ (Invitrogen; Thermo Fisher Scientific, Inc.). RNA purity and concentration were detected using a Nanodrop 2000c spectrophotometer (Thermo Fisher Scientific, Inc.). The reverse transcription was operated using a PrimeScript ${ }^{\mathrm{TM}}$ RT kit (Takara Bio, Inc., Otsu, Japan) according to the manufacturer's protocol. Following reverse transcription, cDNA was diluted with diethy; pyrocarbonate at 1:2 and premixed. Subsequently, $1 \mu \mathrm{l}$ cDNA, primers, premixed liquid and double-distilled water were mixed into a $20 \mu \mathrm{l}$ volume in PCR tubes. Following centrifugation at $800 \mathrm{x}$ g for $5 \mathrm{~min}$ at $4^{\circ} \mathrm{C}$, PCR amplification was performed under the recommended parameters: 40 cycles of $50^{\circ} \mathrm{C}$ for $5 \mathrm{~min}, 95^{\circ} \mathrm{C}$ for $5 \mathrm{~min}, 95^{\circ} \mathrm{C}$ for $5 \mathrm{sec}, 60^{\circ} \mathrm{C}$ for $15 \mathrm{sec}$ and $72^{\circ} \mathrm{C}$ for $15 \mathrm{sec}$, followed by $94^{\circ} \mathrm{C}$ for $15 \mathrm{sec}$. The fluorescence signals were collected and analyzed. The primer sequences were as follows: HIT000218960 forward, 5-CCACCTACC CATCTGACTTTG-3' and reverse, 5-CCACTATTTCCC ACTGCCTT-3; HMGA2 forward, 5-AGAATCTGGTGC AGGAATGG-3 and reverse, 5-TCGTATTTAGTGTCTCCA GCC-3; and $\beta$-actin forward, 5'-CCTCGCCTTTGCCGA TCC-3' and reverse, 5'-GGATCTTCATGAGGTAGTCAG TC- 3 '. The relative RNA expression levels were analyzed using the $2^{-\Delta \Delta C q}$ method (32).

Western blotting. Cells were washed with PBS, and lysed using radioimmunoprecipitation assay buffer (Beyotime Institute of Biotechnology, Haimen, China) for $10 \mathrm{~min}$ on ice. The BCA method was used for protein quantification. Following centrifugation at $14,000 \mathrm{x}$ g for $15 \mathrm{~min}$ at $4^{\circ} \mathrm{C}$, the supernatant was collected. Proteins $(30 \mu \mathrm{g})$ were separated by SDS-PAGE (12\% gel), and transferred onto a polyvinylidene fluoride membrane (EMD Millipore, Billerica, MA, USA). Membranes were washed with Tris-buffered saline containing Tween-20 (TBST), and blocked with 5\% skimmed milk with TBST at $25^{\circ} \mathrm{C}$ for $1 \mathrm{~h}$. Membranes were then incubated with monoclonal primary antibody at $4^{\circ} \mathrm{C}$ overnight. The following primary antibodies were used: Anti-HMGA2 (1:500; catalog no. ab52039; Abcam, Cambridge, UK) and 
A

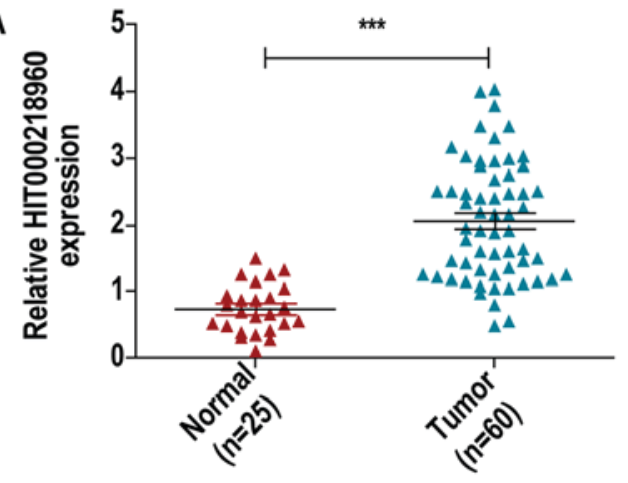

B

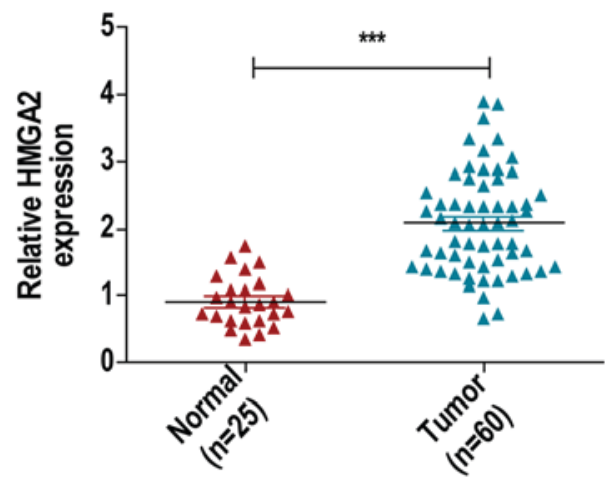

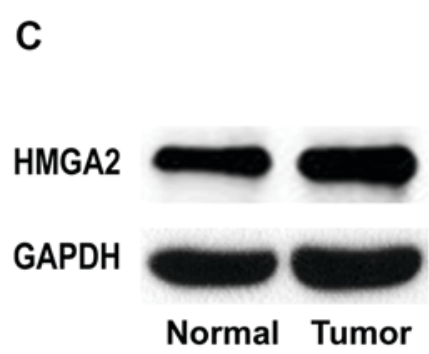
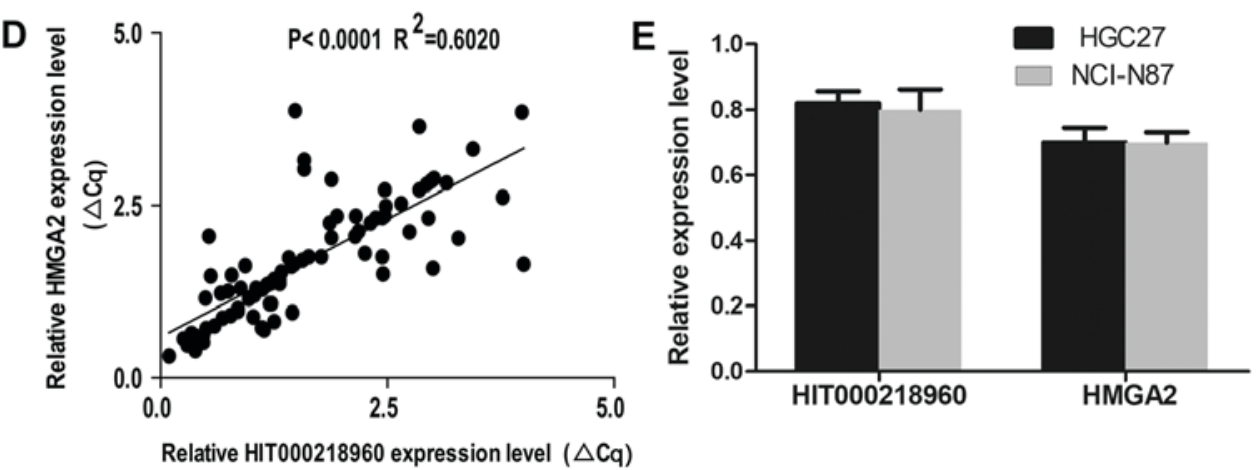

Figure 1. HIT000218960 and HGMA2 are overexpressed in GC tissues. (A) The HIT000218960 expression level is significantly higher in the 60 GC tissues compared with in the 25 normal gastric tissues according to RT-qPCR results. (B) The HMGA2 expression level is significantly higher in the 60 GC tissues compared with in the 25 normal gastric tissues according to RT-qPCR results. (C) Western blotting indicates that the HMGA2 expression level is markedly higher in GC tissues compared with in normal gastric tissues. (D) Positive correlation between HIT000218960 and HMGA2 expression levels. (E) HIT000218960 and HMGA2 expression levels in HGC27 and NCI-N87 cells. ${ }^{* * *} \mathrm{P}<0.001$. GC, gastric cancer; HMGA2, high-mobility group AT-hook 2; RT-qPCR, reverse transcription-quantitative polymerase chain reaction.

anti-GAPDH (1:500; catalog no. ab9485; Abcam). Membranes were washed with TBST for $30 \mathrm{~min}$ and incubated at $25^{\circ} \mathrm{C}$ for $1 \mathrm{~h}$ with goat anti-rabbit $\operatorname{IgG} \mathrm{H} \& \mathrm{~L}$ secondary antibody (1:1,000; catalog no. ab6940; Abcam). Enhanced chemiluminescence reagent (Thermo Fisher Scientific, Inc.) was used to detect the signal on the membrane.

Cell Counting Kit-8 (CCK-8) assay. Cells (2,000 cells/well) were seeded in 96-well plates. CCK-8 reagent (10 $\mu \mathrm{l}$; Dojindo Molecular Technologies, Inc., Kumamoto, Japan) was added to each well at 4, 24, 48, 72, 96 and $120 \mathrm{~h}$ after seeding for $1.5 \mathrm{~h}$. Each group comprised 6 wells and contained a blank control. The absorbance value of each well was determined using a microplate reader at a wavelength of $490 \mathrm{~nm}$.

Migration assay. A Transwell assay was performed to determine the cell migratory ability. HCG2 and NCI-N87 cells $\left(25 \times 10^{4}\right)$ were digested, suspended in serum-free RPMI-1640 medium and seeded onto the upper non-Matrigel-coated chamber with a membrane pore size of 8.0- $\mu \mathrm{m}$ (Corning, Inc., Corning, NY, USA). The lower chamber was filled with $500 \mu 1$ RPMI-1640 medium containing 10\% FBS. After $2 \mathrm{~h}$, cells that had invaded the lower chamber were fixed for 20 min with $95 \%$ ethanol at room temperature, stained for $20 \mathrm{~min}$ with $0.5 \%$ crystal violet at room temperature and washed with water. Subsequently, images were captured with a light microscope (magnification, $\mathrm{x} 400$ ) and cells were counted.
Statistical analysis. SPSS software (version 22.0; IBM Corp., Armonk, NY, USA) was used to analyze data. Images were edited with GraphPad Prism 6.0 software (GraphPad Software, Inc., La Jolla, CA, USA). Clinical characteristics were evaluated using a $\chi^{2}$ test. Student's t-test was applied for comparing data between two groups. Comparison between multiple groups was performed using one-way analysis of variance followed a least significant difference multiple-range post hoc test. Pearson correlation analysis was used to determine the correlation between one dependent variable and single independent variable. $\mathrm{P}<0.05$ was considered to indicate a statistically significant difference.

\section{Results}

Expression levels of HIT000218960 and HMGA2 in GC tissue samples. A total of $60 \mathrm{GC}$ tissue samples and 25 normal control samples were collected. Expression levels of HIT000218960 and HMGA 2 in the two groups were detected using RT-qPCR. The results indicated that HIT000218960 and HMGA2 expression levels were significantly higher in GC samples compared with in control samples ( $\mathrm{P}<0.001$; Fig. $1 \mathrm{~A}$ and $\mathrm{B}$, respectively). In addition, western blotting revealed that HMGA2 protein expression was higher in GC samples compared with in control samples (Fig. 1C). Further analysis indicated a positive correlation between HIT000218960 and HMGA2 expression levels in $\mathrm{GC}$ tissues $\left(\mathrm{P}<0.001 ; \mathrm{R}^{2}=0.6020\right.$; Fig. 1D). However, there 
Table I. Association between HIT000218960 expression level and clinical characteristics of patients with gastric cancer (n=60).

HIT000218960 expression level

Clinical characteristic

$\mathrm{n}$

Low $(n=30)$

High $(\mathrm{n}=30)$

P-value

\section{Age, years}

$\leq 60$

14

17

0.4383

$>60$

29

16

13

Sex

Male

0.3006

Female

Tumor size, cm

$\leq 3$

$>3$

TNM stage

I-II

III-IV

Lymph node metastasis

\begin{tabular}{llrrr} 
Negative & 27 & 18 & 9 & $0.0195^{\text {a }}$ \\
Positive & 33 & 12 & 21 & \\
\hline
\end{tabular}

${ }^{\mathrm{a}} \mathrm{P}<0.05$. TNM, Tumor-Node-Metastasis.
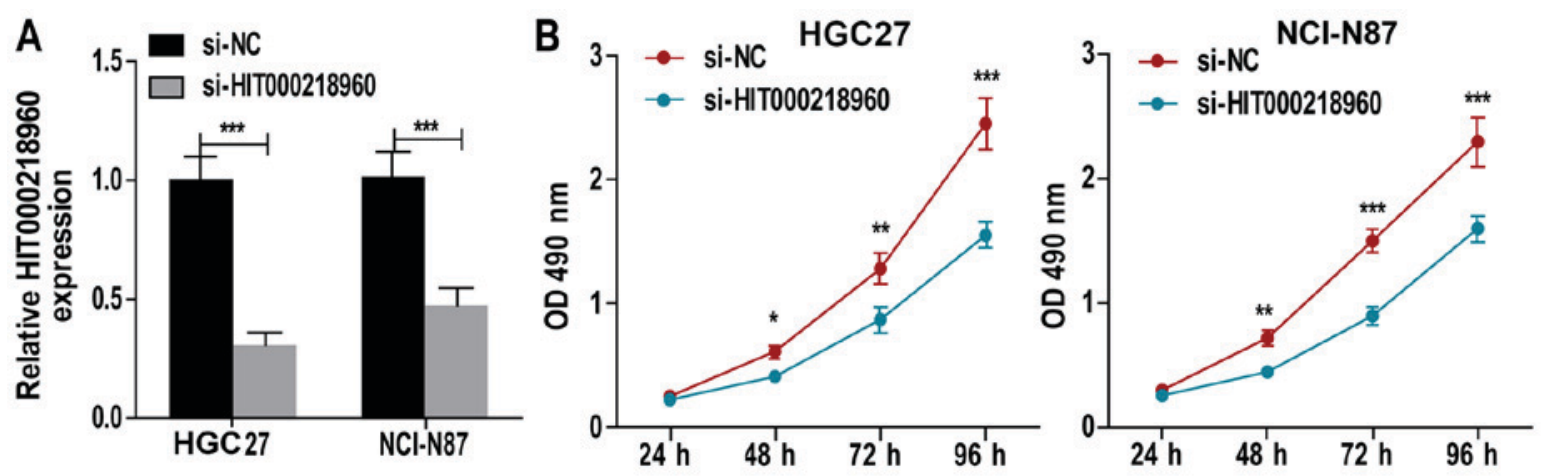

C
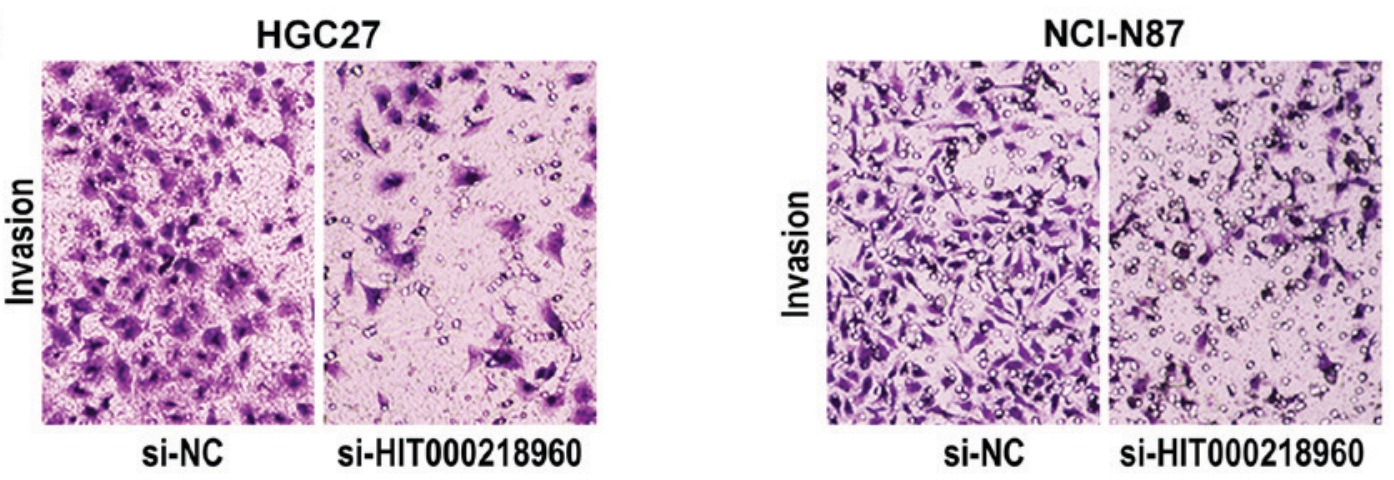

Figure 2. HIT000218960 silencing suppresses GC cell proliferative and migratory ability. (A) Reverse transcription-quantitative polymerase chain reaction results indicated that si-HIT000218960 transfection significantly inhibits the HIT000218960 expression level in GC cell lines. (B) A Cell Counting Kit-8 assay indicated that HGC27 and NCI-N87 cancer cell proliferation is significantly decreased following HIT000218960 silencing. (C) A Transwell assay indicated that the migratory ability of HGC27 and NCI-N87 cell lines decreased following HIT000218960 silencing. Magnification, $\mathrm{x} 400 .{ }^{*} \mathrm{P}<0.05,{ }^{* * *} \mathrm{P}<0.01,{ }^{* * * *} \mathrm{P}<0.001$ vs. si-NC. GC, gastric cancer; NC, negative control; OD, optical density; si, small interfering.

was no difference in HIT000218960 and HMGA2 expression levels between HGC27 and NCI-N87 cell lines (Fig. 1E).
Association between HIT000218960 and clinical characteristics of patients with GC. To clarify the function of 


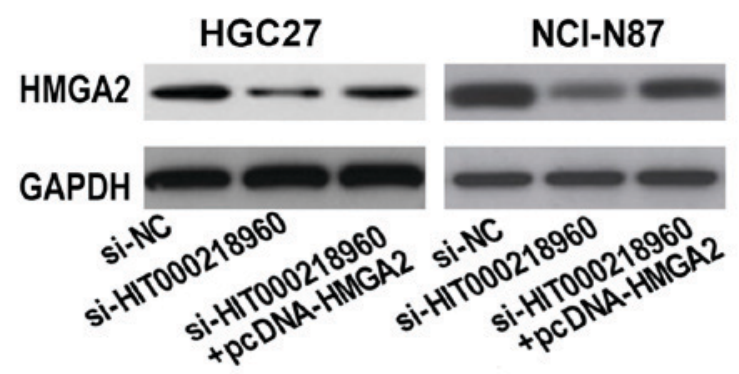

B
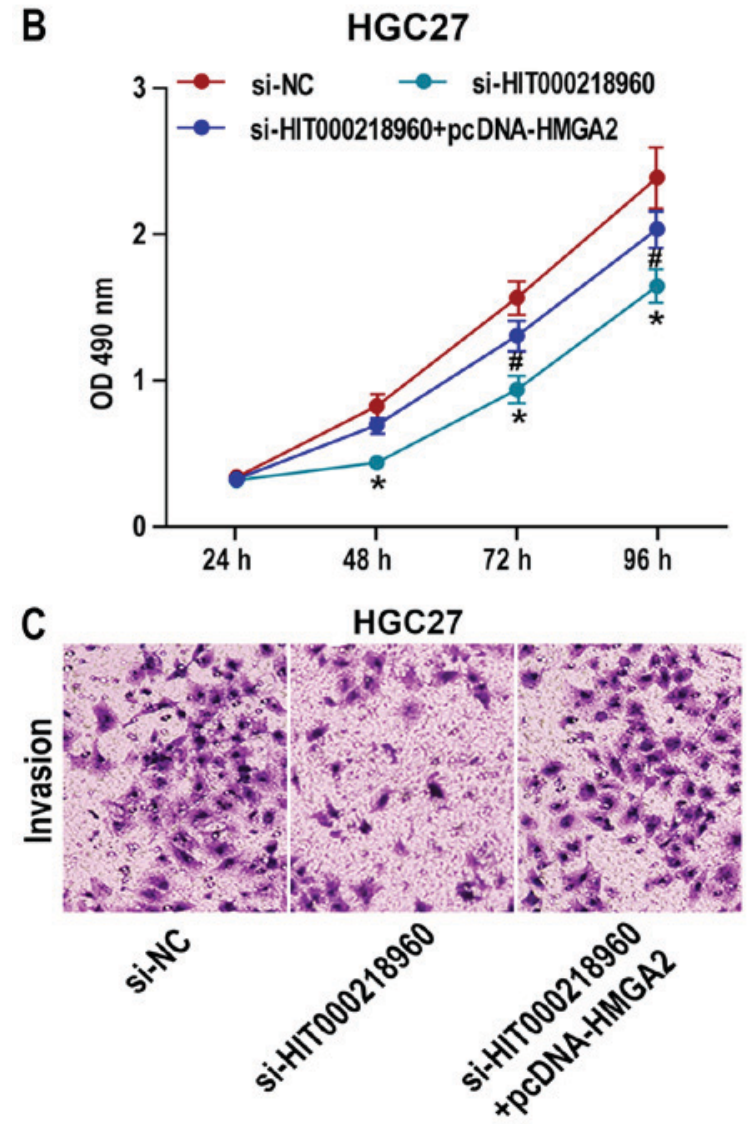

$\mathrm{NCl}-\mathrm{N} 87$
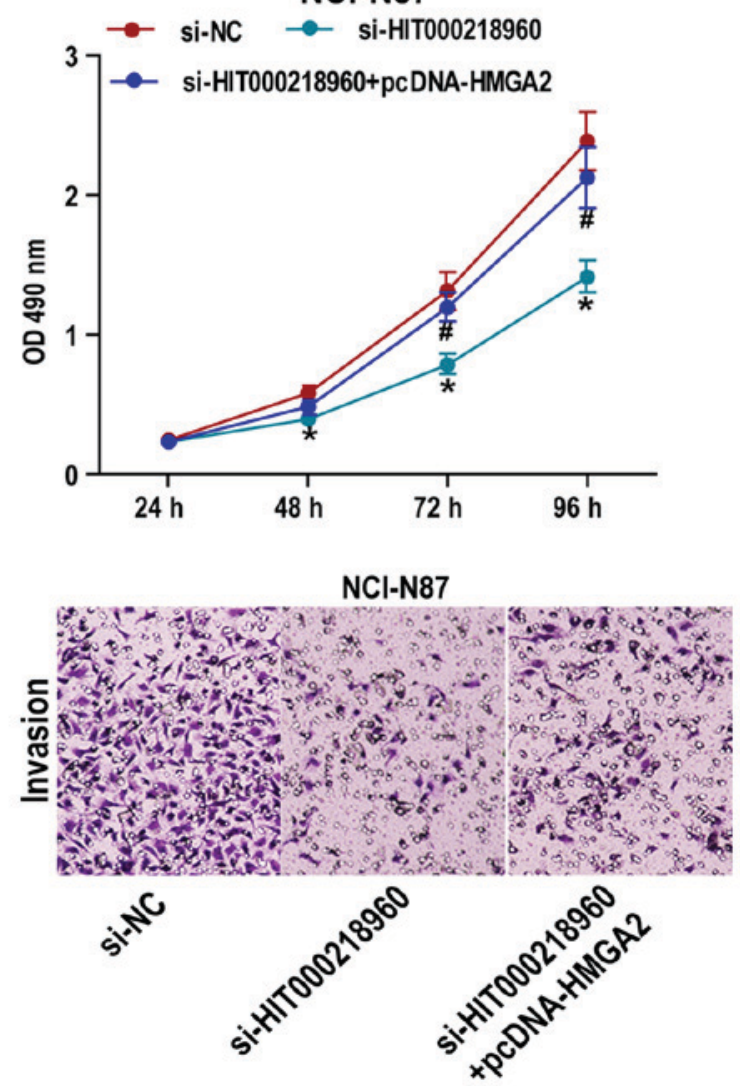

Figure 3. HMGA2 regulates the effect of HIT000218960 on GC cell proliferation and migration. (A) HIT000218960 silencing markedly decreased the HMGA2 protein level in HGC27 and NCI-N87 cell lines, and transfection with pcDNA-HMGA2 increased the expression of HMGA2. (B) A Cell Counting Kit-8 assay indicated that HMGA2 overexpression reverses the si-HIT000218960-induced decrease in proliferation in HGC27 and NCI-N87 cell lines. (C) A Transwell assay indicated that HMGA2 overexpression reverses the si-HIT000218960-induced decrease in the migratory capacity of HGC27 and NCI-N87 cells. Magnification, $\mathrm{x} 400 .{ }^{\text {"P }}<0.05$ vs. si-NC; ${ }^{~} \mathrm{P}<0.05$ vs. si-HIT000218960. GC, gastric cancer; HMGA2, high-mobility group AT-hook 2; NC, negative control; OD, optical density; si, small interfering.

HIT000218960 in GC tissue, the association between the clinical characteristics and HIT000218960 expression levels of the 60 patients with GC was analyzed. Patients were divided into lower-expression and higher-expression groups according to the median level of HIT000218960. Patients with an expression level $\geq 2.1$ were placed in the higher-expression group and patients with an expression level $<2.1$ were placed in the lower-expression group, and each group consisted of 30 patients (Table I). Analysis using a $\chi^{2}$ test indicated that the tumor size of patients in the higher-expression group was significantly larger compared with those in the lower-expression group $(\mathrm{P}=0.0019)$. In addition, compared with the lower-expression group, the rate of advanced TNM staging $(\mathrm{P}=0.0008)$ and the incidence of lymph node metastasis $(\mathrm{P}=0.0195)$ were higher in the higher-expression group $(\mathrm{P}=0.0008$ and $\mathrm{P}=0.0195$, respectively). These data suggested that the level of HIT000218960 expression was associated with tumor size, TNM stage and lymph node metastasis in patients with GC.

HIT000218960 silencing inhibits HGC27 and NCI-N87 cell proliferation and migration. RT-qPCR analysis indicated that si-HIT000218960 transfection significantly decreased HIT000218960 expression levels in HGC27 and NCI-N87 cell lines $(\mathrm{P}<0.001$; Fig. 2A). CCK-8 assay demonstrated that HGC27 and NCI-N87 cell proliferation was significantly decreased following si-HIT000218960 transfection compared with si-NC ( $\mathrm{P}<0.001$; Fig. 2B). The results of the Transwell assay indicated that HIT000218960 silencing decreased HGC27 and NCI-N87 cell migratory ability (Fig. 2C). These results suggested that decreasing HIT000218960 expression decreased GC cell proliferative and migratory ability. 
HMGA2 mediates the regulatory effect of HIT000218960 on GC cell proliferation and migration. Western blotting indicated that HIT000218960 knockdown induced HMGA2 downregulation in HGC27 and NCI-N87 cells, whereas pcDNA-HMGA2 reversed this phenomenon (Fig. 3A). In order to further clarify the association between HIT000218960 and HMGA2, HGC27 and NCI-N87 cells were transfected with si-NC, si-HIT000218960 or si-HIT000218960+pcDNA-HMGA2. A CCK-8 assay revealed that HMGA2 overexpression reversed the si-HIT000218960-induced decrease in cell proliferation (Fig. 3B) and migration (Fig. 3C) of HGC27 and NCI-N87 cell lines. These results suggested that HIT000218960 may promote the proliferative and migratory ability of $\mathrm{HGC} 27$ and NCI-N87 cells through HMGA2 upregulation.

\section{Discussion}

GC is a common gastrointestinal cancer in China, which leads to high mortality and low 5-year survival rates. The treatment of GC is essentially based on conventional surgery; however, there is currently a lack of research on the mechanisms of occurrence and development of GC, compared with other gastrointestinal malignancies. It has been reported that lncRNAs are involved in numerous biological processes, and that dysregulation of RNA expression is associated with various types of disease, including cancer. Subsequently, studies have identified the underlying mechanisms of lncRNA in cancer. For example, the antisense non-coding RNA in the INK4 locus and HOX transcript antisense RNA can determine chromatin localization by recruiting chromatin-modifying complexes and can regulate histone modifications $(33,34)$. To the best of our knowledge, only a few studies on HIT000218960 have been performed. A recent study reported that HIT000218960 is highly expressed in thyroid carcinoma tissues, and that HIT000218960 inhibition induces a decrease in the papillary thyroid carcinoma cell proliferative, migratory ability and invasive capacity (29). They also reported for the first time that HMGA2 regulates HIT00021896 expression. However, the effect of HIT000218960 in GC has not been yet reported. Further investigation on the mechanism of action of HIT000218960 in GC is therefore required. The aim of the present study was to investigate the effect of HIT000218960 in GC. The results demonstrated for the first time that HIT000218960 expression level was significantly higher in GC tissues compared with normal tissues, and that HIT000218960 knockdown inhibited HGC27 and NCI-N87 cell proliferative and migratory ability.

A previous study reported that HMGA2 overexpression is closely associated with the occurrence and development of GC. Motoyama et al (33) observed that HMGA2 expression level in cancerous tissue was high in 110 patients with gastric cancer and was positively correlated with infiltration depth and lymph node metastasis. In addition, follow-up analysis revealed that patients with a high HMGA2 expression level had a shorter survival time compared with that of healthy subjects. This was consistent with the results from the present study, where HMGA2 expression was higher in $\mathrm{GC}$ tissues compared with in normal tissues. In addition, HIT000218960 expression level was positively correlated with HMGA2 expression level in GC tissues. HMGA2, which is a non-histone protein present in the nucleus, can change chromosome structure and regulate the expression of various target genes (15). HMGA2 is now considered to be a oncogene (27). However, the underlying molecular mechanism of HMGA2 in tumors is not fully understood and may be associated with activation or translocation of certain oncogenes. Motoyama et al (33) reported that in the transforming growth factor $\beta$ signaling pathway, Smad upregulates its downstream target genes, including Snail, Twist1 and Snail family transcriptional repressor 2 through HMGA2 and functions as an epithelial (E-)cadherin transcription inhibitor. This results in downregulation of E-cadherin expression and loss of intercellular polarity, which induces EMT. Currently, the association between HMGA2 and the clinical characteristics of patients is being investigated to determine whether HMGA2 can be used to distinguish benign from malignant tumors. For example, a high HMGA2 expression level is associated with $\mathrm{N}$ staging, 2 -year metastasis and poor prognosis in patients with nasopharyngeal carcinoma $(35,36)$. In the present study, the expression levels of HIT000218960 and HMGA2 were associated with the clinical characteristics of patients with GC. These results suggested that HIT000218960 may serve a tumorigenic role by upregulating HMGA2 expression.

In conclusion, the results of the present study indicated that HIT000218960 and HMGA2 were significantly overexpressed in GC tissues and HGC27 an NCI-N87 cell lines. HIT000218960 and HMGA2 expression levels were positively correlated in GC tissues, and the HIT000218960 expression level in patients with GC was associated with tumor size, TNM stage and lymph node metastasis. In addition, HIT000218960 promoted the HGC27 and NCI-N87 cell proliferative and migratory capacity by upregulating HMGA2 expression.

\section{Acknowledgements}

Not applicable.

\section{Funding}

No funding was received.

\section{Availability of data and materials}

All data generated or analyzed during this study are included in this published article.

\section{Authors' contributions}

LS and SR designed the study and performed the experiments. JY, PW and MS collected the data. JY and PW analyzed the data. LS and SR prepared the manuscript. All authors read and approved the final manuscript.

\section{Ethics approval and consent to participate}

This study was approved by the Ethics Committee of The First Affiliated Hospital of Zhejiang Chinese Medical University 
(Hangzhou, China). All subjects and/or guardians provided written informed consent.

\section{Patients consent for publication}

Not applicable.

\section{Competing interests}

The authors declare that they have no competing interests.

\section{References}

1. Chen W, Zheng R, Baade PD, Zhang S, Zeng H, Bray F, Jemal A, $\mathrm{Yu}$ XQ and He J: Cancer statistics in China, 2015. CA Cancer J Clin 66: 115-132, 2016.

2. Ferlay J, Soerjomataram I, Dikshit R, Eser S, Mathers C, Rebelo M, Parkin DM, Forman D and Bray F: Cancer incidence and mortality worldwide: Sources, methods and major patterns in GLOBOCAN 2012. Int J Cancer 136: E359-E386, 2015.

3. Ilson DH: Adjuvant treatment for gastric cancer: Too much is not enough. Lancet Oncol 15: 788-789, 2014.

4. Bagcchi S: Radiotherapy for testicular cancer increases gastric cancer risk. Lancet Oncol 15: e593, 2014.

5. Konishi H, Ichikawa D, Komatsu S, Shiozaki A, Tsujiura M, Takeshita H, Morimura R, Nagata H, Arita T, Kawaguchi T, et al: Detection of gastric cancer-associated microRNAs on microRNA microarray comparing pre- and post-operative plasma. $\mathrm{Br}$ J Cancer 106: 740-747, 2012.

6. Zirtiloglu A, Cil I, Mehmet VF and Tural D: What are the optimal treatment modalities according to age group in gastric cancer patients? J Buon 22: 312-319, 2017.

7. Spizzo R, Almeida MI, Colombatti A and Calin GA: Long non-coding RNAs and cancer: A new frontier of translational research? Oncogene 31: 4577-4587, 2012.

8. Batista PJ and Chang HY: Long noncoding RNAs: Cellular address codes in development and disease. Cell 152: 1298-1307, 2013.

9. Xia Y, Yan Z, Wan Y, Wei S, Bi Y, Zhao J, Liu J, Liao DJ and Huang H. Knockdown of long noncoding RNA GHET1 inhibits cellcycle progression and invasion of gastric cancer cells. Mol Med Rep 18: 3375-3381, 2018.

10. Lv Z, Zhang Y, Yu X, Lin Y and Ge Y: The function of long non-coding RNA MT1JP in the development and progression of gastric cancer. Pathol Res Pract 214: 1218-1223, 2018.

11. Zhu C, Ma J, Li Y, Zhang Y and Da M: Low expression of long noncoding RNA MT1JP is associated with poor overall survival in gastric cancer patients: Protocol for meta-analysis. Medicine (Baltimore) 97: e10394, 2018.

12. Shi $X$, Wang $X$ and Hua Y: LncRNA GACAT1 promotes gastric cancer cell growth, invasion and migration by regulating miR-149-mediated of ZBTB2 and SP1. J Cancer 9: 3715-3722, 2018.

13. Yang F, Bi J, Xue X, Zheng L, Zhi K, Hua J and Fang G: Up-regulated long non-coding RNA H19 contributes to proliferation of gastric cancer cells. Febs J 279: 3159-3165, 2012.

14. Liu G, Xiang T, Wu QF and Wang WX: Curcumin suppresses the proliferation of gastric cancer cells by downregulating H19. Oncol Lett 12: 5156-5162, 2016.

15. Yang F, Xue X, Bi J, Zheng L, Zhi K, Gu Y and Fang G: Long noncoding RNA CCAT1, which could be activated by c-Myc, promotes the progression of gastric carcinoma. J Cancer Res Clin Oncol 139: 437-445, 2013.

16. Henriksen J, Stabell M, Meza-Zepeda LA, Lauvrak SA, Kassem $\mathrm{M}$ and Myklebost O: Identification of target genes for wild type and truncated HMGA2 in mesenchymal stem-like cells. BMC Cancer 10: 329, 2010.

17. Sun T, Zhao N, Zhao XL, Gu Q, Zhang SW, Che N, Wang XH, Du J, Liu YX and Sun BC: Expression and functional significance of Twist1 in hepatocellular carcinoma: Its role in vasculogenic mimicry. Hepatology 51: 545-556, 2010.

18. Wagenblast E, Soto M, Gutierrez-Angel S, Hartl CA, Gable AL, Maceli AR, Erard N, Williams AM, Kim SY, Dickopf S, et al: A model of breast cancer heterogeneity reveals vascular mimicry as a driver of metastasis. Nature 520: 358-362, 2015.
19. Williamson SC, Metcalf RL, Trapani F, Mohan S, Antonello J, Abbott B, Leong HS, Chester CP, Simms N, Polanski R, et al: Vasculogenic mimicry in small cell lung cancer. Nat Commun 7: $13322,2016$.

20. Wang JY, Sun T, Zhao XL, Zhang SW, Zhang DF, Gu Q, Wang XH, Zhao N, Qie S and Sun BC: Functional significance of VEGF-a in human ovarian carcinoma: Role in vasculogenic mimicry. Cancer Biol Ther 7: 758-766, 2008.

21. Baeten CI, Hillen F, Pauwels P, de Bruine AP and Baeten CG: Prognostic role of vasculogenic mimicry in colorectal cancer. Dis Colon Rectum 52: 2028-2035, 2009.

22. Zhong X, Liu X, Li Y, Cheng M, Wang W, Tian K, Mu L, Zeng T, Liu Y, Jiang X, et al: HMGA2 sustains self-renewal and invasiveness of glioma-initiating cells. Oncotarget 7: 44365-44380, 2016.

23. Fujikane R, Komori K, Sekiguchi M and Hidaka M: Function of high-mobility group A proteins in the DNA damage signaling for the induction of apoptosis. Sci Rep 6: 31714, 2016.

24. Yu H, Lim HH, Tjokro NO, Sathiyanathan P, Natarajan S, Chew TW, Klonisch T, Goodman SD, Surana U and Droge P: Chaperoning HMGA2 protein protects stalled replication forks in stem and cancer cells. Cell Rep 6: 684-697, 2014.

25. Tan EJ, Thuault S, Caja L, Carletti T, Heldin $\mathrm{CH}$ and Moustakas A: Regulation of transcription factor twist expression by the DNA architectural protein high mobility group A2 during epithelial-to-mesenchymal transition. J Biol Chem 287: 7134-7145, 2012.

26. Tan EJ, Kahata K, Idas O, Thuault S, Heldin CH and Moustakas A: The high mobility group A2 protein epigenetically silences the Cdh1 gene during epithelial-to-mesenchymal transition. Nucleic Acids Res 43: 162-178, 2015.

27. Li Y, Zhao Z, Xu C, Zhou Z, Zhu Z and You T: HMGA2 induces transcription factor Slug expression to promote epithelial-to-mesenchymal transition and contributes to colon cancer progression. Cancer Lett 355: 130-140, 2014.

28. Singh I, Mehta A, Contreras A, Boettger T, Carraro G, Wheeler M, Cabrera-Fuentes HA, Bellusci S, Seeger W, Braun T and Barreto G: Hmga2 is required for canonical WNT signaling during lung development. BMC Biol 12: 21, 2014.

29. Li T, Yang XD, Ye CX, Shen ZL, Yang Y, Wang B, Guo P, Gao ZD, Ye YJ, Jiang KW and Wang S: Long noncoding RNA HIT000218960 promotes papillary thyroid cancer oncogenesis and tumor progression by upregulating the expression of high mobility group AT-hook 2 (HMGA2) gene. Cell Cycle 16: 224-231, 2017.

30. Liu C, Ren L, Wang Y, Liu Y and Xiao J: The interaction between RACK1 and WEE1 regulates the growth of gastric cancer cell line HGC27. Oncol Lett 14: 4784-4792, 2017.

31. Kawai S, Kato S, Imai H, Okada Y and Ishioka C: Suppression of FUT1 attenuates cell proliferation in the HER2-overexpressing cancer cell line NCI-N87. Oncol Rep 29: 13-20, 2013.

32. Livak KJ and Schmittgen TD: Analysis of relative gene expression data using real-time quantitative PCR and the $2-\Delta \Delta \mathrm{Ct}$ method. Methods 25: 402-408, 2001.

33. Motoyama K, Inoue H, Nakamura Y, Uetake H, Sugihara K and Mori M: Clinical significance of high mobility group A2 in human gastric cancer and its relationship to let-7 microRNA family. Clin Cancer Res 14: 2334-2340, 2008.

34. Tsai MC, Manor O, Wan Y, Mosammaparast N, Wang JK, Lan F, Shi Y, Segal E and Chang HY: Long noncoding RNA as modular scaffold of histone modification complexes. Science 329: 689-693, 2010.

35. Xia YY, Yin L, Tian H, Guo WJ, Jiang N, Jiang XS, Wu J, Chen M, Wu JZ and He X: HMGA2 is associated with epithelial-mesenchymal transition and can predict poor prognosis in nasopharyngeal carcinoma. Onco Targets Ther 8: 169-176, 2015.

36. Xia YY, Yin L, Jiang N, Guo WJ, Tian H, Jiang XS, Wu J, Chen M, Wu JZ and He X: Downregulating HMGA2 attenuates epithelial-mesenchymal transition-induced invasion and migration in nasopharyngeal cancer cells. Biochem Biophys Res Commun 463: 357-363, 2015.

This work is licensed under a Creative Commons Attribution-NonCommercial-NoDerivatives 4.0 International (CC BY-NC-ND 4.0) License. 